\section{Improved Newton-Type Algorithm for Adaptive Implementation of Pisarenko's Harmonic Retrieval Method and Its Convergence Analysis}

George Mathew, Soura Dasgupta, and Vellenki U. Reddy

\begin{abstract}
Pisarenko's harmonic retrieval (PHR) method is probably the first eigenstructure based algorithm for estimating the frequencies of sinusoids corrupted by additive white noise. To develop an adaptive implementation of the PHR method, one group of authors has proposed a least-squares type recursive algorithm. In their algorithm, they made approximations for both gradient and Hessian. In this paper, we derive an improved algorithm, where we use exact gradient and a different approximation for the Hessian and analyze its convergence rigorously. Specifically, we provide a proof for the local convergence and detailed arguments supporting the local instability of undesired stationary points. Computer simulations are used to verify the convergence performance of the new algorithm. Its performance is substantially better than that exhibited by its counterpart, especially at low SNR's.
\end{abstract}

\section{INTRODUCTION}

Estimation of frequencies of sinusoids corrupted with white noise (additive) is a problem of importance in many applications of signal processing. "Super-resolution" spectral estimation techniques are best suited for this signal model. Pisarenko's harmonic retrieval (PHR) method [1] was perhaps the first of this kind. It involves determining the eigenvector corresponding to the minimum eigenvalue of the covariance matrix of the observed process.

Let the observed data $x(n)$ consist of the sum of $P$ real sinusoids in additive white noise of variance $\sigma^{2}$ and $\mathbf{R}_{x}$ be its asymptotic autocorrelation matrix of size $N \times N(N \geq 2 P+1)$. Suppose the $N$ eigenvalues of $\mathbf{R}_{x}$, in decreasing order of magnitude, are

$$
\gamma_{1} \geq \gamma_{2} \geq \cdots \geq \gamma_{2 P}>\gamma_{2 P+1}=\cdots=\gamma_{N}=\sigma^{2}
$$

and the corresponding orthonormal eigenvectors are $\mathbf{q}_{1}, \cdots, \mathbf{q}_{N}$. Then, it is one of the last $N-2 P$ eigenvectors (corresponding to the $(N-2 P)$ repeated minimum eigenvalues) or any point in the subspace they span that is what the PHR method uses. These eigenvectors will be hereafter referred to as minimum eigenvectors.

Recognizing that a minimum eigenvector is the solution of a constrained minimization problem, Thompson [3] developed an adaptive version of the PHR method using a constrained gradient search procedure. Convergence properties of his algorithm were studied by Larimore [4]. Later, Reddy et al. [2] restated this problem into an unconstrained nonlinear minimization framework and developed a Newton-type recursive algorithm. They used approximations for both the gradient and Hessian of the underlying cost function.

In this paper, we first present an improved version of the algorithm of [2], which incorporates the exact gradient and a different approximation for the Hessian. This leads to an algorithm whose convergence performance is significantly better than that of its counterpart in [2], especially at low SNR's. A key contribution of the paper is the convergence analysis given in Sections III and IV. We show

Manuscript received February 6, 1992; revised February 18, 1993. The associate editor coordinating the review of this paper and approving it for publication was $\mathrm{Dr}$. James Zeidler.

G. Mathew and V. U. Reddy are with the Department of Electrical Communication Engineering, Indian Institute of Science, Bangalore, India.

S. Dasgupta is with the Department of Electrical and Computer Engineering The University of Iowa, Iowa City, IA 52242

IEEE Log Number 9214180 in Section III under mild assumptions that if the initial estimate is "sufficiently close" to the subspace spanned by $\mathbf{q}_{2 P+1}, \cdots, \mathbf{q}_{N}$, the algorithm converges to this subspace. Regarding the behavior of the algorithm in a global context, we give detailed arguments to establish the instability of the undesired stationary points (i.e., $\left.\mathbf{q}_{i}, i \in\{1, \cdots, 2 P\}\right)$.

A brief review of the approximate Newton algorithm of [2] and the derivation of the improved version are given in Section II. Section III presents the local convergence proof and Section IV provides arguments supporting the local instability of undesired stationary subspaces. Section V gives some simulation results and finally, Section VI concludes.

\section{IMPROVED NEWTON-TYPE ALGORITHM}

The cost function used in [2] is

$$
V(\mathbf{a}, t)=\frac{1}{2} \sum_{s=1}^{t} e^{2}(s)
$$

where $e(s)=\overline{\mathbf{a}}^{T} \mathbf{x}(s), \overline{\mathbf{a}}=\mathbf{a} /\|\mathbf{a}\|$ with $\mathbf{a}$ being the coefficient vector of size $N \times 1$ and $\mathbf{x}(s)=[x(s), x(s-1), \cdots, x(s-N+1)]^{T}$, the data vector at $s$ th instant. The Newton-type algorithm proposed in [2] is given by

$$
\begin{aligned}
\mathbf{a}(k) & =\|\mathbf{a}(k-1)\|(\overline{\mathbf{a}}(k-1)-\mathbf{P}(k) \zeta(k) e(k)) \\
\mathbf{P}(k) & =\left[\sum_{s=1}^{k} \zeta(s) \zeta^{T}(s)\right]^{-1} \\
& =\mathbf{P}(k-1)-\frac{\mathbf{P}(k-1) \zeta(k) \zeta^{T}(k) \mathbf{P}(k-1)}{1+\zeta^{T}(k) \mathbf{P}(k-1) \zeta(k)} \\
\zeta(k) & =\mathbf{x}(k)-\overline{\mathbf{a}}(k-1) e(k) \quad \text { and } \\
e(k) & =\overline{\mathbf{a}}(k-1)^{T} \mathbf{x}(k) .
\end{aligned}
$$

In arriving at (3), they used the instantaneous gradient of $V$ and the Hessian of $V$ was approximated so as to facilitate a recursive updating for the inverse of the Hessian, $\mathbf{P}(k)$. However, the recursion for $\mathbf{P}(k)$ makes use of all the earlier coefficient vectors through $\mathbf{P}(k-i), i=1, \cdots, k-1$. This is inconsistent in that the gradient and Hessian evaluated using the coefficient vector at the present instant need to be used in the Newton update.

We now present the improved version. Consider the following cost function

$$
V^{\prime}(\mathbf{a}, t)=\frac{V(\mathbf{a}, t)}{t}=\frac{1}{2 t} \sum_{s=1}^{t} e^{2}(s) .
$$

With $e(s)=\overline{\mathbf{a}}^{T} \mathbf{x}(s)$, we can express the gradient and Hessian of $V^{\prime \prime}$ as

$$
\begin{aligned}
\mathbf{g}_{2}= & \frac{1}{\|\mathbf{a}\|}\left[\mathbf{R}(t) \overline{\mathbf{a}}-\left(\overline{\mathbf{a}}^{T} \mathbf{R}(t) \overline{\mathbf{a}}\right) \cdot \overline{\mathbf{a}}\right] \\
\mathbf{H}_{2}= & \frac{1}{\|\mathbf{a}\|^{2}}\left[\mathbf{R}(t)+4\left(\overline{\mathbf{a}}^{T} \mathbf{R}(t) \overline{\mathbf{a}}\right) \overline{\mathbf{a}}^{T}-2 \mathbf{R}(t) \overline{\mathbf{a}}^{T}\right. \\
& \left.-2 \overline{\mathbf{a}}^{T} \mathbf{R}(t)-\left(\overline{\mathbf{a}}^{T} \mathbf{R}(t) \overline{\mathbf{a}}\right) \cdot \mathbf{I}_{N}\right]
\end{aligned}
$$

where $\mathbf{R}(t)=1 / t \Sigma_{s=1}^{t} \mathbf{x}(s) \mathbf{x}^{T}(s)$. For reasons mentioned already, we approximate the Hessian as below

$$
\mathbf{H}_{2} \approx \hat{\mathbf{H}}_{2}=\frac{1}{\|\mathbf{a}\|^{2}}\left[\mathbf{R}(t)+4\left(\overline{\mathbf{a}}^{T} \mathbf{R}(t) \overline{\mathbf{a}}\right) \overline{\mathbf{a}}^{T}\right] .
$$


Now, using (7) and (9) in the Newton update algorithm, we obtain the following algorithm

$$
\mathbf{a}(k)=l(k) \mathbf{R}^{-1}(k) \mathbf{a}(k-1)
$$

where $l(k), \mathbf{R}(k)$, and $\mathbf{R}^{-1}(k)$ are enumerated in (11)-(13), which appear at the bottom of the page. Note that this algorithm not only uses the exact gradient but also both the Hessian and gradient are evaluated using the coefficient vector at the present instant. However this algorithm requires an additional $2.5 N^{2}$ multiplications compared to the earlier [2].

\section{Convergence Analysis of the NeW Algorithm}

In this section, we provide a proof of local convergence of the recursive algorithm, given in (10)-(13). The convergence analysis is based on the following two assumptions.

1. Ergodicity: The underlying process $\{x(n)\}$ is assumed to be ergodic. That is, $\exists \mathbf{R}_{x}$ such that $\mathbf{R}^{-1}(n)$ of (13) obeys

$$
\lim _{n \rightarrow \infty} \mathbf{R}^{-1}(n)=\mathbf{R}_{x}^{-1} \text {. }
$$

2. Richness: The data are assumed to be rich. That is, $\exists \alpha_{1}>0$ and $\alpha_{2}>0$ such that

$$
\alpha_{1} \mathbf{I}_{N} \leq \mathbf{R}^{-1}(n) \leq \alpha_{2} \mathbf{I}_{N} \quad \forall n \geq 0 .
$$

Let $\mathbf{Q}=\left[\mathbf{q}_{1}, \cdots, \mathbf{q}_{N}\right]$ and $\Gamma=\operatorname{diag}\left[\gamma_{1}, \gamma_{2}, \cdots, \gamma_{N}\right]$. In the analysis of convergence, it is important to specify a precise quantification of the distance between $\mathbf{a}(k)$ and the subspace of minimum eigenvectors of $\mathbf{R}_{x}$. Define

$$
\begin{aligned}
\mathbf{b}(k) & =\mathbf{Q}^{T} \mathbf{a}(k) \quad \text { and } \\
f(k) & =\frac{\left\|\left[b_{1}(k), \cdots, b_{2 P}(k)\right]\right\|_{\infty}}{\left\|\left[b_{2 P+1}(k), \cdots, b_{N}(k)\right]\right\|_{\infty}} .
\end{aligned}
$$

Clearly, $f(k)=0$ implies $\mathbf{a}(k)$ is in the desired subspace. Premultiplying (10) with $\mathbf{Q}^{T}$, we obtain

$$
\mathbf{b}(k)=l(k)\left[\boldsymbol{\Gamma}^{-1}+\boldsymbol{\Psi}(k)\right] \mathbf{b}(k-1)
$$

where $\boldsymbol{\Psi}(k)=\mathbf{Q}^{T}\left[\mathbf{R}^{-1}(k)-\mathbf{R}_{x}^{-1}\right] \mathbf{Q}$. The following Lemma will prove useful.

Lemma: Under (14) and (15), the quantities in (17) have the following properties

1. $\frac{1}{\alpha_{2}} \leq l(k) \leq \frac{1}{\alpha_{1}} \quad \forall k$

2. $\lim _{k \rightarrow \infty} \boldsymbol{\Psi}(k)=0$

3. There exists $k_{0}$ and $\delta_{4}, \delta_{5}, \delta_{6}$ (all are positive scalars) such that $\forall k \geq k_{0}$ (with $\psi_{i j}(k)$ denoting the $i j$ th element of $\boldsymbol{\Psi}(k)$ )

$$
\begin{array}{ll}
\text { a. } & \frac{\frac{1}{\gamma_{2 P}}+\psi_{2 P, 2 P}(k)}{\frac{1}{\gamma_{2 P+1}}+\psi_{2 P+1,2 P+1}(k)}<1-\delta_{4} \\
\text { b. } & \delta_{5}<l(k)\left[\frac{1}{\gamma_{1}}+\psi_{11}(k)\right] \\
& l(k)\left[\frac{1}{\gamma_{2 P}}+\psi_{2 P, 2 P}(k)\right]
\end{array}
$$$$
\text { b. } \quad \delta_{5}<l(k)\left[\frac{1}{\gamma_{1}}+\psi_{11}(k)\right] \leq \cdots \leq
$$

$$
\begin{aligned}
& \text { c. } \quad l(k)\left[\frac{1}{\gamma_{i}}+\psi_{i i}(k)\right]<\delta_{6} \quad \forall i \in\{1, \cdots, N\} \\
& \text { d. }\left[\frac{1}{\gamma_{2 P}}+\psi_{2 P, 2 P}(k)\right]<\left[\frac{1}{\gamma_{i}}+\psi_{i i}(k)\right] \forall i>2 P(21 \mathrm{c}) \\
& \text { e. } \lim _{k \rightarrow \infty}\left|\psi_{i i}(k)-\psi_{j j}(k)\right|=0 \quad \forall i, j \in\{2 P+ \\
& 1, \cdots, N\} \\
& \text { f. }\left|\psi_{i j}(k)\right|<\frac{\delta_{5} \delta_{4}}{8 N} \quad \forall i \neq j .
\end{aligned}
$$

Now, observe that the underlying process governing the behavior of $f(k)$ in (16) is such (see (15) and (18)) that for every $k_{0}, \exists \epsilon_{6}$ such that

$$
\left|f\left(k_{0}\right)\right|<1 \quad \text { whenever } \quad|f(0)|<\epsilon_{6} .
$$

Then, we have the following main result (proved in the appendix).

Theorem There exists $\epsilon_{6}$ such that with $|f(0)|<\epsilon_{6}$

$$
\lim _{k \rightarrow \infty} f(k)=0 \text {. }
$$

IV. LOCAL INSTABILITy OF THE UNDESIRED SUBSPACES

In Section III, we have shown how the desired subspace, i.e., the space spanned by the minimum eigenvectors, is locally stable. In this section, we examine the behavior of the algorithm in a global context. Observe the following. Even in the ideal case of $\mathbf{R}(k)=\mathbf{R}_{x}$ for all $k$, one cannot guarantee global convergence since every eigenvector of $\mathbf{R}_{x}$ is a stationary point of this algorithm. Further, all of the additional "stationary subspaces" are locally unstable. Hence, in practice, even if one starts exactly on any of these undesired subspaces, as $\mathbf{R}(k) \neq \mathbf{R}_{x}$ for all $k$, the trajectories will leave these subspaces because of local instability and eventually be attracted to the desired subspace. Simulations given in Section $\mathrm{V}$ confirm this behavior.

To support these points, consider the case where $\mathbf{R}(k)=\mathbf{R}_{x} \forall k \geq$ 0 . Then, $\boldsymbol{\Psi}(k)=\mathbf{0}$ and $\mathbf{b}(k)=l(k) \Gamma^{-1} \mathbf{b}(k-1)$. Observe, if $\mathbf{a}(k-1)=\alpha \mathbf{q}_{i}$ for some $\alpha \neq 0$, then $\mathbf{a}(k)=\alpha \mathbf{q}_{i}$. Thus, the subspace $\alpha \mathbf{q}_{i}$ is an invariant subspace for any $i$.

Now, suppose $\mathbf{a}(k-1)$ is close to $\alpha \mathbf{q}_{i}$ for $i \in\{1, \cdots, 2 P\}$ (i.e. $\left.b_{i}(k) \gg b_{j}(k) \forall i \neq j\right)$ with at least one of $b_{2 P+1}(k), \cdots, b_{N}(k)$ being nonzero. This results in $l(k) \approx \gamma_{i}$ and $b_{j}(k) \cong\left(\gamma_{i} / \gamma_{j}\right) b_{j}(k-$ 1) $\forall j$. Thus, if $\gamma_{i}<\gamma_{j}, b_{j}(k)$ decreases. More importantly, if $\gamma_{i}>\gamma_{j}$ (i.e., $j \geq 2 P+1), b_{j}(k)$ increases exponentially at a rate faster than that experienced by $b_{j}(k)$ for $j \leq 2 P$. Thus, the algorithm forces the trajectories to move away from the vicinity of $\alpha \mathbf{q}_{i}$ for $i \in\{1, \cdots, 2 P\}$, toward the desired subspace.

\section{Simulation Results}

The data samples were generated from

$$
x(n)=\beta \sin (0.4 \pi n)+\beta \sin (0.48 \pi n+\theta)+v(n) .
$$

The amplitude $\beta$ was chosen to give the desired SNR, defined as $10 \log _{10}\left(\beta^{2} / 2\right)$. The initial phase $\theta$ (uniformly distributed in $[-\pi, \pi]$ ) and $v(n)$, a zero-mean white noise of unit variance, were varied from trial to trial of the Monte Carlo simulations.

$$
\begin{aligned}
l(k) & =\frac{5 \cdot\|\mathbf{a}(k-1)\|^{2}}{\frac{\|\mathbf{a}(k-1)\|^{4}}{\mathbf{a}^{T}(k-1) \mathbf{R}(k) \mathbf{a}(k-1)}+4 \mathbf{a}^{T}(k-1) \mathbf{R}^{-1}(k) \mathbf{a}(k-1)} \\
\mathbf{R}(k) & =\frac{k-1}{k} \mathbf{R}(k-1)+\frac{1}{k} \mathbf{x}(k) \mathbf{x}^{T}(k) \\
\mathbf{R}^{-1}(k) & =\frac{k}{k-1}\left[\mathbf{R}^{-1}(k-1)-\frac{\mathbf{R}^{-1}(k-1) \mathbf{x}(k) \mathbf{x}^{T}(k) \mathbf{R}^{-1}(k-1)}{k-1+\mathbf{x}^{T}(k) \mathbf{R}^{-1}(k-1) \mathbf{x}(k)}\right], \quad \forall k \geq 2 .
\end{aligned}
$$




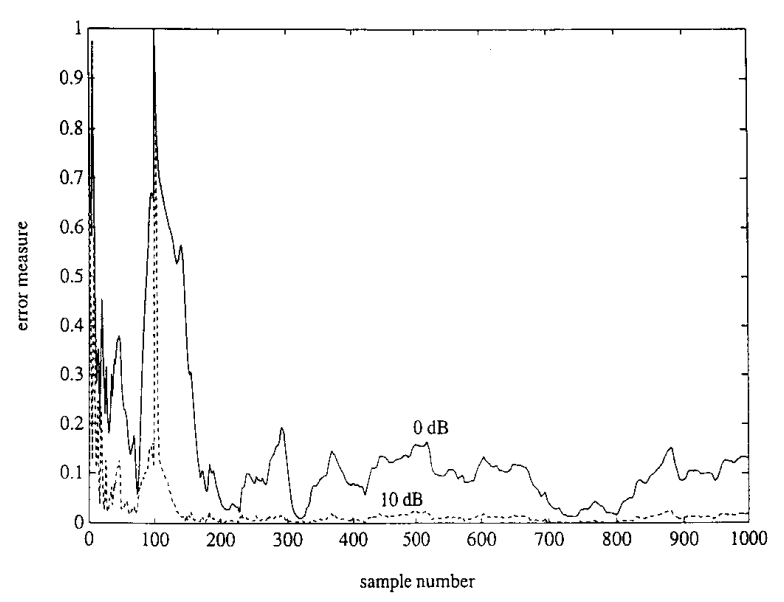

Fig. 1. Convergence performance of the new algorithm with reinitialization to the largest eigenvector at 100 th data sample.

Let $\hat{\mathbf{e}}(k)$ denote the estimated eigenvector (normatized to unit norm). Then, the performance measure $(E)$ was chosen as the norm of that part of $\hat{\mathbf{e}}(k)$ that lies outside the desired subspace.

In our convergence proof, we assumed an appropriate initialization for $\mathbf{a}(k)$. However, the following simulations illustrate that this assumption is not essential for the algorithm to converge (in line with the discussion in Section IV). Fixing $N$ at 6 , we ran the new algorithm up to 99 data samples. Then, we forced $\mathbf{a}(100)$ to $\mathbf{q}_{1}$ and left the algorithm to run further. Fig. 1 shows the plots of error measure $E$ for two different values of SNR, $0 \mathrm{~dB}$ and $10 \mathrm{~dB}$ (for the same data realization). The error measure in the case of $0 \mathrm{~dB}$ SNR initially fluctuates wildly, whereas for $10 \mathrm{~dB}$ SNR, it falls off quickly. At the 100th data sample, it becomes the maximum possible because of the reinitialization of $a(100)$ to $\mathbf{q}_{1}$. Thereafter, it falls and fluctuates about a small value, implying that the vector $\mathbf{a}$ is moving away from $\mathbf{q}_{1}$ toward a minimum eigenvector. The larger fluctuations and residual error measure in the case of $0 \mathrm{~dB}$ SNR are due to the well-known result on tradeoff between the data size and the SNR; if the SNR is larger, the convergence of the data covariance matrix close to its asymptotic value is quicker.

We repeated this with various data realizations and also with reinitialization at different sample points. In all the cases, the algorithm essentially behaved as in Fig. 1. We also observed that moving away from the reinitialized point was faster when the SNR was higher. This is again due to the quicker convergence of $\mathbf{R}(k)$ close to its asymptotic value when the SNR is higher. Thus, these simulations show that appropriate initialization of the algorithm is not essential for the algorithm to converge.

To see how the new algorithm performs (on average) compared to the earlier version [2], we conducted the following simulations. Fixing $N=6$, we applied both of them to the same 100 different data realizations, computed the average error measure at each data sample, and plotted the results in Fig. 2(a)-(b). These figures correspond to $0 \mathrm{~dB}$ and $10 \mathrm{~dB}$ SNR's, respectively. Note from the plots that the new algorithm converges significantly faster than the earlier version, particularly at low SNR. As stated in [2], at high SNR, the approximations made in the algorithm of [2] are good in the neighborhood of the desired subspace. Consequently, the convergence performance of their algorithm is much less affected by the approximations at high SNR. This is why the performance edge of the new algorithm drops as the SNR increases.

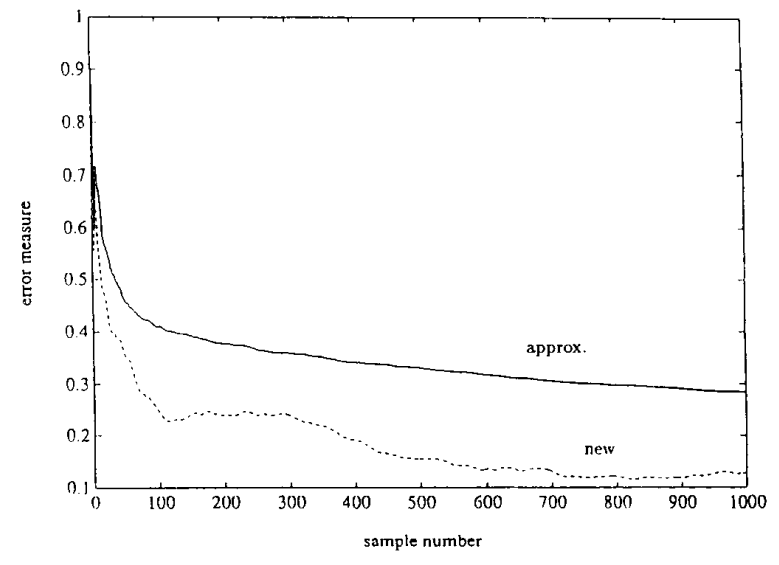

(a)

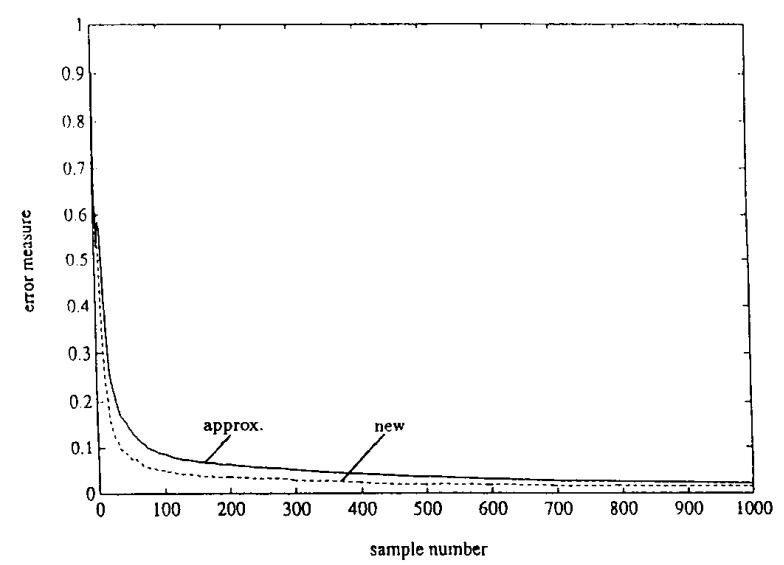

(b)

Fig. 2. Comparison of the convergence performance of the two algorithms, new and approximate versions (averaged over 100 trials). (a) For $0 \mathrm{~dB}$ SNR. (b) For $10 \mathrm{~dB}$ SNR.

\section{CONCLUDING REMARKS}

In this correspondence, we derived an improved version of the Newton-type algorithm of Reddy et al. [2] for adaptively seeking the minimum eigenvector of the asymptotic covariance matrix of the data consisting of sinusoids in white noise. More importantly, we developed a proof of convergence of the new algorithm. Though we assumed an appropriate initialization of the algorithm in the development of the convergence proof, our experience with extensive simulations shows that such initialization is not essential for the algorithm to converge.

We compared the convergence performance of the new algorithm to that of [2]. Simulations show that the new algorithm converges significantly faster than that in [2], particularly in the case of low SNR.

Though we motivated the algorithm for the case of sinusoids in white noise, it is equally applicable to the case where the asymptotic covariance matrix is symmetric and positive definite.

\section{APPENDIX}

In this appendix, we prove the theorem of Section III. But first, we develop the following preliminary result. 
Lemma B: Consider a matrix $\mathbf{D}$ of size $n \times n$. Suppose $\forall i, \exists\left|\epsilon_{i}\right|<$ $\epsilon$, such that

$$
d_{i i}=\lambda+\epsilon_{i} \quad \text { and } \quad\left|d_{i j}\right|<\epsilon \quad \forall i \neq j .
$$

Then, $\forall \mathbf{y} \in \mathbf{R}^{n}$

$$
\|\mathbf{D y}\|_{\infty} \geq[|\lambda|-n \epsilon]\|\mathbf{y}\|_{\infty} .
$$

Proof: Expressing $\mathbf{D}=\lambda \mathbf{I}_{n}+\overline{\mathbf{D}}$, we get from (26) that $\|\overline{\mathbf{D}}\|_{\infty}<n \epsilon$. Then, it follows that

$$
\begin{aligned}
\|\mathbf{D} \mathbf{y}\|_{\infty} & \geq|\lambda|\|\mathbf{y}\|_{\infty}-\|\overline{\mathbf{D}} \mathbf{y}\|_{\infty} \\
& \geq|\lambda|\|\mathbf{y}\|_{\infty}-n \epsilon\|\mathbf{y}\|_{\infty} .
\end{aligned}
$$

Proof of the Theorem: Let $\Phi(k)=l(k)\left[\Gamma^{-1}+\boldsymbol{\Psi}(k)\right]$. Partition $\mathbf{b}(k)$ as

$$
\mathbf{b}(k)=\left[\mathbf{w}^{T}(k), \mathbf{y}^{T}(k)\right]^{T}
$$

where $\mathbf{w}(k)=\left[b_{1}(k), \cdots, b_{r}(k)\right]^{T}$ and $\mathbf{y}(k)=$ $\left[b_{r+1}(k), \cdots, b_{N}(k)\right]^{T}$ with $r=2 P$. Similarly, partition $\boldsymbol{\Phi}(k)$ into $\boldsymbol{\Phi}_{w y}, \boldsymbol{\Phi}_{w w}, \boldsymbol{\Phi}_{y y}$, and $\boldsymbol{\Phi}_{y w}$ so that (17) becomes

$$
\left[\begin{array}{l}
\mathbf{w}(k+1) \\
\mathbf{y}(k+1)
\end{array}\right]=\left[\begin{array}{ll}
\boldsymbol{\Phi}_{w w}(k) & \boldsymbol{\Phi}_{w y}(k) \\
\boldsymbol{\Phi}_{y w}(k) & \boldsymbol{\Phi}_{y y}(k)
\end{array}\right]\left[\begin{array}{l}
\mathbf{w}(k) \\
\mathbf{y}(k)
\end{array}\right] \text {. }
$$

Then, we have the following

$$
\begin{aligned}
f(k+1) & =\frac{\|\mathbf{w}(k+1)\|_{\infty}}{\|\mathbf{y}(k+1)\|_{\infty}} \\
& \leq \frac{\left\|\boldsymbol{\Phi}_{w w}(k)\right\|_{\infty}\|\mathbf{w}(k)\|_{\infty}+\left\|\boldsymbol{\Phi}_{w y}(k)\right\|_{\infty}\|\mathbf{y}(k)\|_{\infty}}{\left\|\boldsymbol{\Phi}_{y y}(k) \mathbf{y}(k)\right\|_{\infty}-\left\|\boldsymbol{\Phi}_{y w}(k) \mathbf{w}(k)\right\|_{\infty}} .
\end{aligned}
$$

Because of (19) and (21d), we can write $\forall k \geq k_{0}$

$$
\begin{aligned}
\left|\phi_{i j}(k)\right|<\epsilon_{1}(k) & \forall i \neq j \\
\left|\phi_{i i}(k)-\phi_{j j}(k)\right|<\epsilon_{1}(k) & \forall i, j \in\{r+1, \cdots, N\}
\end{aligned}
$$

where $\epsilon_{1}(k)$ is such that $0<\epsilon_{1}(k)<\epsilon_{1}$ and $\lim _{k \rightarrow \infty} \epsilon_{1}(k)=0$. Denoting $\phi_{i i}(k)=\phi_{i}(k)$ and using Lemma B, (31), (21) and (32), for all $k \geq k_{0}$, (30) can be expressed as

$$
\begin{aligned}
f(k+1) \leq & \frac{1}{\phi_{N}(k)-N \epsilon_{1}(k)(1+f(k))} \\
& \cdot\left[\phi_{r}(k) f(k)+N \epsilon_{1}(k)\right] .
\end{aligned}
$$

Now, choose $\epsilon_{1}<\delta_{5} \delta_{4} / 8 N$. Then, if $f(k)<1$, using (20) and (21), we can reduce (33) to

$$
\begin{aligned}
f(k+1) & \leq \frac{1}{\left(1-\frac{\delta_{4}}{4}\right)}\left[\left(1-\delta_{4}\right) f(k)+\frac{N \epsilon_{1}(k)}{\phi_{N}(k)}\right] \\
& \leq \frac{1}{\left(1-\frac{\delta_{4}}{4}\right)}\left[\left(1-\delta_{4}\right) f(k)+\frac{\delta_{4}}{8}\right] .
\end{aligned}
$$

Thus, in view of (23), (35) implies that $f(k)<1 \forall k \geq k_{0}$. Now, consider (34). Since $\phi_{N}(k)>\delta_{5}$, it can be written as

$$
f(k+1) \leq \frac{\left(1-\delta_{4}\right)}{\left(1-\frac{\delta_{4}}{4}\right)} f(k)+\frac{N}{\delta_{5}\left(1-\frac{\delta_{4}}{4}\right)} \epsilon_{1}(k) .
$$

As $\left(1-\delta_{4}\right)<\left(1-\delta_{4} / 4\right)$ and $\lim _{k-\infty} \epsilon_{1}(k)=0$, we can conclude from (36) that

$$
\lim _{k \rightarrow \infty} f(k)=0
$$

\section{REFERENCES}

[1] V. F. Pisarenko, "The retrieval of harmonics by a covariance function," Geophys. J. Royal Astron. Soc., pp. 347-366, 1973.

[2] V. U. Reddy, B. Egardt, and T. Kailath, "Least squares type algorithm for adaptive implementation of Pisarenko's harmonic retrieval method," IEEE Trans. Acoust. Speech Signal Processing, vol. ASSP-30, pp. 399-405, June 1982.

[3] P. A. Thompson, "An adaptive spectral analysis technique for unbiased frequency estimation in the presence of white noise," in Proc. 13th Asilomar Conf. Circuits, Syst., Comput. (Pacific Grove, CA), 1979, pp. 529-533.

[4] M. G. Larimore, "Adaptive convergence of spectral estimation based on Pisarenko's harmonic retrieval," IEEE Trans. Acoust. Speech Signal Processing, vol. ASSP-31, pp. 955-962, Aug. 1983.

\section{Design of Linear-Phase IIR Filters from FIR Specifications}

M. F. Fahmy, Y. M. Yassin, G. Abdel-Raheem, and N. El-Gayed

Abstract-A simple method is presented for the characterization of a stable IIR filter matching a finite portion of the impulse response and autocorrelation coefficients of a given FIR filter. It is shown that the problem is reduced to the solution of a set of linear equations derived using the impulse response and autocorrelation data. The method is characterized by its computational simplicity and is illustrated by some examples to show its superior performance when compared to the existing methods.

\section{INTRODUCTION}

Several methods have been proposed to approximate an FIR filter by an IIR equivalent [1]-[3]. Nearly all these methods are nonoptimum in the sense of achieving the desired approximation but with increased degrees. Recently, two methods for FIR to IIR transformation have been described, [4]-[5]. Both methods rely on singular value decomposition (SVD) of the state covariance matrix of the original FIR to get the reduced-order IIR filter. Consequently, many computations are required, especially for large-order FIR. Compuations have also revealed that for a given FIR, both methods yield the same IIR filter in most cases.

In this paper, we propose a method requiring only the solution of a set of linear equations for the characterization of a stable IIR filter matching a finite portion of the impulse response and autocorrelation coefficients of a given FIR filter. It is shown that the necessary conditions required in order that these data represent a stable IIR filter can always be met. Illustrative examples are given to show that the proposed method requires a far less number of computations and in most cases yields an improved response. In short, it competes very favorably with the existing approaches.

Manuscript received May 5, 1992; revised November 17, 1992. The associate editor coordinating the review of thispaper and approving it for publication was Prof. Tamal Bose.

The authors are with the Department of Electrical and Electronic Engineering, University of Assuit, Assuit, Egypt.

IEEE Log Number 9214174. 